\title{
Histochemical Changes in the Skin Following Local Application of Cortisol or Prednisolone'
}

\author{
BURTON L. BAKER AND LEOPOLDO F. MONTES 2,3 \\ Department of Anatomy, University of Michigan Medical School, \\ Ann Arbor, Michigan
}

The prolonged daily application of adrenocortical extract or cortisone induces profound histological alterations in the skin of rats. These changes are limited to the area of treatment (Baker and Whitaker, '48; Castor and Baker, '50) and are particularly impressive in males. They include (a) cessation in growth of hair, (b) thinning of epidermis, with reduction in size and flattening of epidermal cells, (c) thinning of the dermis with disappearance of subcutaneous fat, (d) compaction of dermal collagenous fibers, (e) injury to cellular components of the dermis, especially those in a superficial location, and (f) reduction in size of sebaceous glands. In addition, adrenocortical hormones by local action facilitate the spreading action of hyaluronidase (Hayes, Reed and Baker, '50), inhibit granuloma formation (Meier et al., '50; Baker and Whitaker, '50; Baker, '54), and suppress the inflammatory reaction. These studies demonstrated the remarkable capacity of adrenocortical compounds to alter normal histology of cutaneous tissues by direct action. Few attempts have been made to define more precisely the influence of adrenocortical hormones on the chemistry of cells in situ when this action is exerted locally and, thus, is not complicated by the profound metabolic alterations which these agents elicit after systemic administration.

The recent refinement of histochemical technics for the localization of oxidative and hydrolytic enzymes within cells indicates that these procedures might be used effectively in combination with local treatment with adrenocortical steroids to gain information concerning the manner in which steroid hormones modify cellular chemistry. The introduction of nitro-blue tetrazolium in particular has made possible a much more precise localization of oxidative enzymes (Nachlas et al., '57). Hence, the objective of this investigation is to observe some of the cytochemical changes which occur in various types of cutaneous cells following the application of cortisol to the skin. Since little information is available concerning the local histological effects of analogs of cortisol, prednisolone ( $\Delta^{1}$-dehydrohydrocortisone) was studied also.

\section{MATERIALS AND METHODS}

At the beginning of the experiment, hair was clipped from the dorsum of the neck of adult male Long-Evans or SpragueDawley rats in an area lying between the midlateral lines of the neck and extending longitudinally from the base of the ears to the middle of the scapulae. Seventyeight Long-Evans rats with an average body weight of $314 \mathrm{gm}$ were divided equally between cortisol- and alcoholtreated groups in 6 experiments, alcohol being used as solvent for the hormones. Eighteen Long-Evans and 6 SpragueDawley rats were arranged similarly for study of prednisolone.

Crystalline cortisol ${ }^{4}$ and prednisolone were dissolved in $25 \%$ ethanol at a concentration of $1 \mathrm{mg}$ per $\mathrm{ml}$. One-tenth milliliter of the solvent $25 \%$ ethanol or of the hormone was applied twice daily to an area just caudal to the right ear according

\footnotetext{
${ }^{1}$ Supported in part by research grants from the National Institutes of Health (A-2841) and The Upjohn Company.

2 The technical assistance of Mrs. Charlene Koth, Mrs. Pamela Vanderhyde and Mrs. Zelda Klapper is appreciated.

3 Present address: Leopoldo F. Montes, M.D., Ave. Libertador 15082, Acassuso, Buenos Aires, Argentina.

4 Our appreciation is extended to Dr. Elmer Alpert of Merck and Company, Rahway, New Jersey, for supplying generous quantities of cortisol and prednisolone.
} 
to the procedure described previously (Whitaker and Baker, '48). Graphic records of the hair growth pattern were made and the hair clipped at weekly intervals. Suppression of hair growth made it possible to evaluate the effectiveness of the treatment. Treatment was continued for 61 to 140 days.

At termination of the experiment, samples of skin were removed from both the treated area on the right side of the neck and from the left nontreated side. Thus, each animal receiving the hormone served as its own control. Excision of skin samples from similar areas on alcohol-treated rats made possible the detection of any effects which might have been induced by the solvent alone.

From each area under study, one strip of skin was fixed in chilled picric-alcoholformalin (PAF) and sections of it were stained subsequently with periodic acidleucofuchsin (PAS) and counterstained with Harris' hematoxylin, methylene blue (citric acid-sodium phosphate buffer, $\mathrm{pH}$ 5.8) or azure II. Another strip was frozen at approximately $-80^{\circ} \mathrm{C}$ and sectioned at $6 \mu$ in a Cryostat at $-18^{\circ} \mathrm{C}$. The sections were flattened on coverslips by warming and air-dried for 10 minutes before incubation. They were then stained for alkaline phosphatase (AP) (Gomori, '52), nonspecific esterase (NSE) (Gomori, '52) using alpha naphthyl acetate as substrate and Diazo Red $\mathrm{RC}$ as the coupling agent, succinic dehydrogenase (SD) (Farber and Bueding, '56), diphosphopyridine nucleotide diaphorase (DPND) (Scarpelli, Hess and Pearse, '58), lactic dehydogenase (LD) (Hess, Scarpelli and Pearse, '58) and cytochrome c oxidase (CO) (Burstone, '59a). All incubations were carried out at $37^{\circ} \mathrm{C}$ without stirring. Sections for $\mathrm{SD}$ were incubated for 30 to 60 minutes; for LD and DPND, 5 to 30 minutes; for $C O$ and for NSE, 20 minutes; and for $\mathrm{AP}, 4$ hours. Following incubation, fat was removed from the sections prepared for NSE by immersion in acetone for 10 minutes. The DPND and LD preparations were immersed for 10 minutes in $10 \%$ ethanol to remove excess dye. Before mounting with glychrogel, all sections except those for SD were fixed for 10 minutes in $10 \%$ buffered formalin.
Control sections were run with all reactions with the specific substrate being omitted. In all cases no staining occurred. In addition $0.003 \mathrm{M}$ oxalic acid was used as an inhibitor for DPND by immersing the section in it for 10 minutes at $5^{\circ} \mathrm{C}$ before incubation in the substrate.

\section{OBSERVATIONS}

In comparing skin which had been treated with a hormone or alcohol with nontreated specimens, special attention was given to the status of hair growth in the control sample since the thickness of the epidermis is often greater in an area of active hair growth as compared with inactive regions. This correlation has been described for the mouse (Chase et al., '53). Since adrenocortical steroids suppress growth of hair in the area of treatment, it was necessary to compare treated skin with those control areas in which hair was not growing.

Microscopic sections stained with PAS and hematoxylin or azure II revealed that prednisolone (figs. 1 and 2) elicited responses identical with those of cortisone or cortisol as summarized previously.

In the following summary of the cutaneous changes induced by hormones, comparisons are made between the right treated side of the neck and the left nontreated side. Treatment with alcohol alone did not modify the skin significantly. LD and $\mathrm{CO}$ were studied only after cortisol; the other enzymes were observed after both cortisol and prednisolone therapy.

Epidermis. In all samples of skin from areas not treated with hormone, the NSE procedure revealed granular dye deposits throughout the stratum germinativum (fig. 6). Especially prominent were scattered cells in the basal layer which were intensely reactive. The cells extended also into the outer epithelial sheath of the hair follicle (fig. 4). They were more common in the pigmented Long-Evans strain than in albino Sprague-Dawley rats. Study of sections fixed in PAF failed to reveal the identity of these cells.

$\mathrm{SD}$ and $\mathrm{CO}$ reactions were similar in several respects. The dye deposits were in the form of fine bodies of variable size (fig. 3 ). Both reactions were erratic, being present in some parts of the section and absent 
in others. The stratum germinativum was stained similarly and intensely by the DPND (fig. 10) and LD (fig. 12) procedures. The purple formazan deposits, in the form of granules and rods, were darkest and most concentrated in the basal layer. In larger cells toward the surface they were more diffusely distributed and of a lighter color. Although these deposits assumed the form and concentration of mitochondria, in portions of some basal cells more dye was present than could be accounted for with LD by staining of mitochondria only. In addition, a diffuse light red stain was imparted to the cytoplasm of the deepest cells. No AP was observed in the epidermis.

Following treatment with cortisol or prednisolone, the sites of dye deposit with the NSE procedure were usually more sparsely distributed (fig. 7), which in view of the concurrent reduction in size of epidermal cells indicated a fall in activity per cell. Most impressively, the localization of high activity in isolated cells of the basal layer was no longer present, indicating either elimination of a cell type from the epidermis or a much more profound loss of demonstrable enzyme activity in isolated cells as compared with other epidermal cells.

The epidermal cells in skin treated with adrenocortical hormones usually exhibited a small reduction in the concentration of intracellular formazan with DPND (fig. 11). Because the epidermis was thinner and its constituent cells smaller, the total activity of the epidermis was depressed. The change in LD followed a similar pattern (fig. 13). Because of irregularity in the $S D$ and $C O$ reactions, evaluation of the overall status of these enzymes could not be made. In those specimens which were adequate, the cells of the stratum germinativum showed $\mathrm{SD}$ and $\mathrm{CO}$ activity comparable to that in nontreated areas.

Hair follicles. In skin not treated with hormones, the external root sheath resembled the epidermal stratum germinativum in its response to the various enzyme reactions (figs. 4 and 10 ) with the following exception. The basal epidermal cells which stained intensely for NSE were found only in that part of the sheath above the sebaceous glands. The reactions for
$\mathrm{SD}$ and $\mathrm{CO}$ were obtained more regularly than in epidermis, especially in the external epithelial sheath deep to the sebaceous glands in growing follicles. The epithelial hair papillae were intensely active in AP (fig. 8).

Treatment with cortisol or prednisolone resulted in a profound involution of the external epithelial sheath. In fact, it seemed probable that many follicles disappeared completely since extensive regions observed in microscopic sections of treated skin were devoid of hair follicles. All the oxidative and hydrolytic enzymes studied exhibited a depletion which parallelled the extent of cellular atrophy (fig. 11). Some activity of all enzymes remained as long as sheath cells were present. The epithelial hair papillae were held dormant by hormone treatment. If structurally identifiable, they still revealed the presence of AP.

Sebaceous glands. Sebaceous glands in nontreated skin were stained intensely by the NSE (fig. 4), DPND (fig. 11) and LD procedures. With the latter two methods, variable types of staining were obtained. First, there were fine granular and rodshaped bodies which were probably mitochondria. Second, a diffuse red stain was imparted to the background cytoplasm.

Finally there were large clumps and crystals of stain most of which appeared to be formed by solution of formazan in sebum. The largest clumps were found at the mouth of the gland where formation of secretion was most nearly complete. This conclusion was supported by brief treatment of the section with acetone prior to incubation to remove sebum. These large masses were then absent while the remainder of the staining capacity of the gland was not completely destroyed. With SD small granules were evident peripherally and coarse clumps of dye centrally. The latter may have resulted from accumulation of reduced formazan in sebum. No precise localization was obtained for $\mathrm{CO}$.

In hormone-treated skin the profound atrophy of sebaceous glands inevitably reduced the total amount of SD, DPND and LD activity. However, the small glands which remained still stained with an intensity comparable to that of nontreated skin (fig. 11). The reaction for NSE dif- 
fered from the responses to all other procedures in that a suppression of activity occurred apart from atrophy of the gland. Thus, the glands in treated skin stained less intensely than those from nontreated areas (fig. 5). However, the secretion contained in the gland at its mouth was unchanged in its staining intensity. These observations indicate that hydrolytic activity is present in sebum, that the rate of its formation in sebaceous glands is suppressed by the action of adrenocortical steroids, and that this effect occurs independently of cellular involution. The concentration of AP in the adjacent connective tissue was not altered noticeably (fig. 9).

Dermis. The free cells of dermis in nontreated skin were highly active for NSE, DPND (fig. 10), LD and usually CO. In most preparations $\mathrm{SD}$ was not revealed consistently. Accurate identification of cell types was impossible but the stellate shape of most cells suggested that the majority were fibroblasts. The endothelium of the subepidermal capillaries was active in NSE, DPND (fig. 10) and LD (fig. 12). So much AP was present in the capillary wall that structure was obscured by the cobalt sulfide deposits (fig. 8). The arrector pili muscle was highly active in NSE (fig. 4), SD, CO, DPND (fig. 10) and LD.

Hormone treatment resulted in extensive atrophy and often complete disappearance of free cells in the superficial portion of the dermis (figs. 5 and 11). However, insofar as could be determined from these preparations the enzymes remained as long as cells were present. Enzyme activity was unchanged in the capillary wall (figs. 9 and 11 ) and cells of the arrector pili muscle (figs. 5, 11), although the latter structures were often considerably smaller than in nontreated skin.

\section{DISCUSSION}

\section{The localization of enzymes}

The relationship of the intracellular formazan deposits to mitochondria following reactions which are dependent on mitochondrial enzymes is not entirely clear. SD and CO are recognized generally to be located in mitochondria (Dixon and Webb, '58) and to participate in the succinic oxidase system (Pearse, '60). The formazan resulting from SD activity and the staining by the $\mathrm{CO}$ procedure appeared as fine granules or rods of similar distribution in the epidermis, external sheath of the hair follicle and arrector pili muscle. However, in the dermal connective tissue cells, SD was rarely demonstrated but $\mathrm{CO}$ was regularly present. SD was always revealed in sebaceous glands but only nonspecific diffuse staining was seen with the CO procedure. With SD (Scarpelli and Pearse, '58) and CO (Burstone, '59b) it appears highly probable that the dye is deposited either within or on mitochondria. Certainly the size and number of dye deposits are similar to those of mitochondria in the cells in question.

The distribution and intensity of staining obtained with the DPND and LD reactions were remarkably similar, suggesting that the distribution of the diaphorase may be the limiting factor in the $L D$ reaction. In both cases mitochondria were stained. This is somewhat unexpected with LD because biochemical analysis of cellular fractions indicates that this enzyme is found in the supernatant (Dixon and Webb, '58). However, the DPND reaction is regarded as being superior to the common cytological methods for demonstrating mitochondrial morphology (Scarpelli, Hess and Pearse, '58).

In the epidermis more formazan was deposited in some basal cells with the DPND and LD procedures than could be accounted for by direct staining of mitochondria alone. Furthermore, background cytoplasm was stained with red formazan, especially with the LD technic. The enzymatic significance of this type of staining reaction is not yet understood (Nachlas et al., '57). DPND and LD might be moved from mitochondria to cytoplasm as a result of injury from freezing and thawing. Since evidence derived from biochemical analysis of cell fractions shows that DPND is present in the microsomal fraction (Kuff and Dalton, '59) and LD in the soluble fraction (Dixon and Webb, '58), staining of extramitochondrial cytoplasm would be expected.

Epidermis. The demonstration of SD in the epidermis of the rat with histochemical procedures has proven difficult. 
Pearson ('58) and Rutenburg et al. ('53) did not observe activity, and Padykula ('52) used sections too thick for precise localization. Nachlas et al. ('57) found a weak reaction in the basal layers. In this study SD was present in the stratum germinativum of sections $6 \mu$ thick, the formazan appearing as fine granules. Nevertheless, we were unable to control the reaction sufficiently to consistently demonstrate the enzyme throughout all of the epidermis in a section.

\section{Relation to hormone action}

Available biochemical evidence derived from the analysis of subcellular fractions and intact cells indicates that the action of steroid hormones, including those of the adrenal cortex, is to inhibit oxidation especially if the steroids are applied in high concentrations. Thus, steroid hormones are potent inhibitors of enzymes involved in the oxidation of DPNH (Hayano, Dorfman and Yamada, '50; Yielding and Tomkins, '59). Corticosterone inhibits oxidation of DPNH at the point of cytochrome $\mathrm{c}$ reductase rather than the DPN diaphorase; cortisol acts similarly but is less potent (Jensen, '59). Cortisol appears to suppress the action of DPN-linked mitochondrial dehydrogenases by increasing the permeability of the mitochondrial membrane which results in leaching of DPN from the mitochondria (Gallagher, '58). Generally, succinate oxidation has not been inhibited by cortisol.

These and other observations suggested the possibility that histochemical technics might make possible the detection of interference with oxidative processes by adrenocortical steroids. Yielding and Tomkins ('59) have suggested that the growth inhibiting actions of adrenocortical steroids, which are so dramatically demonstrated by the response of cutaneous tissues, could result in part from the inhibitory action of the hormones on oxidative functions. However, in this study no change was observed in the oxidative enzymes which could be divorced from general atrophy of the tissue in question. The experiment was not designed to reveal whether a change in oxidative enzyme activity preceded structural atrophy. Only in the case of the hydrolytic NSE in the sebaceous gland was a suppression of activity demonstrated apart from involution of the tissue in question.

The destruction of connective tissue cells by local action of cortisol and prednisolone emphasizes the extreme sensitivity of these cells to high levels of adrenocortical hormones as compared with other cellular components of the skin.

\section{SUMMARY}

Nonspecific esterase, alkaline phosphatase, DPN diaphorase, lactic dehydrogenase, succinic dehydrogenase and cytochrome c oxidase were studied in the skin after prolonged local application of cortisol or prednisolone twice daily. Nonspecific esterase was reduced in sebaceous glands. Total DPN diaphorase and lactic dehydrogenase activities were reduced in epidermis coincident with thinning of this structure. These enzymes, in addition to succinic dehydrogenase and cytochrome $c$ oxidase, remained active in the smaller cells of the treated epidermis. Nonspecific esterase, DPN diaphorase, lactic dehydrogenase and cytochrome c oxidase were depleted from connective tissue cells and the external epithelial sheath of the hair follicle as they underwent involution due to hormone action.

\section{LITERATURE CITED}

Baker, B. I. 1954 The connective tissue reaction around implanted pellets of steroid hormones. Anat. Rec., 119: 529-540.

Baker, B. L., and W. L. Whitaker 1948 Growth inhibition in the skin following direct application of adrenal cortical preparations. Ibid., 102: $333-347$.

1950 Interference with wound healing by the local action of adrenocortical steroids. Endocrinology, 46: 544-551.

Burstone, M. S. 1959a New histochemical techniques for the demonstration of tissue oxidase (cytochrome oxidase). J. Histochem. Cytochem., 7: 112-122.

- 1959b Histochemical localization of oxidase activity in the mitochondria of the human heart. Nature (Lond.), 184 (Suppl. 7): 476-477.

Castor, C. W., and B. I. Baker 1950 The local action of adrenocortical steroids on epidermis and connective tissue of the skin. Endocrinology, 47: 234-241.

Chase, H. B., W. Montagna and J. D. Malone 1953 Changes in the skin in relation to the hair growth cycle. Anat. Rec., 116: 75-81.

Dixon, M., and E. C. Webb 1958 Enzymes. Academic Press, Inc., New York. 
Farber, E., and E. Bueding 1956 Histochemical localization of specific oxidative enzymes. V. The dissociation of succinic dehydrogenase from carriers by lipase and the specific histochemical localization of the dehydrogenase with phenazine methosulfate and tetrazolium salts. J. Histochem. Cytochem., 4: 357-362.

Gallagher, C. H. 1958 Effect of hydrocortisone on mitochondrial membrane permeability. Nature, 182: 1315-1316.

Gomori, G. 1952 Microscopic Histochemistry. University of Chicago Press, Chicago.

Hayano, M., R. I. Dorfman and E. Y. Yamada 1950 The inhibition of d-amino acid oxidase by desoxycorticosterone. J. Biol. Chem., 186: 603-614.

Hayes, M. A., T. G. Reed and B. L. Baker 1950 Dermal spreading of hyaluronidase as influenced by prolonged local treatment with adrenal cortical extract. Proc. Soc. Exp. Biol. Med., 75: 361-363.

Hess, R., D. G. Scarpelli and A. G. E. Pearse 1958 The cytochemical localization of oxidative enzymes. II. Pyridine nucleotide-linked dehydrogenases. J. Biophys. Biochem. Cytol., 4: $753-760$.

Jensen, P. K. 1959 Corticosterone inhibition of pyridine nucleotide oxidase from heart sarcosomes. Nature, 184: (Suppl. 7) : 451-452.

Kuff, E, and A. Dalton 1959 Biochemical studies of isolated Golgi membranes. In: Subcellular Particles, T. Hayashi, ed. The Ronald Press Co., New York, p. 114.

Meier, R., W. Schuler and P. Desaulles 1950 Zur Frage des Mechanismus der Hemmung des Bindegewebswachstums durch Cortisone. Experientia, 6: 469-471.
Nachlas, M. M., K-C Tsou, E. de Souza, C-S Cheng and A. M. Seligman 1957 Cytochemical demonstration of succinic dehydrogenase by the use of a new p-nitrophenyl substituted ditetra. zole. J. Histochem. Cytochem., 5: 420-436.

Padykula, H. A. 1952 The localization of succinic dehydrogenase in tissue sections of the rat. Am. J. Anat., 91: 107-146.

Pearse, A. G. E. 1960 Histochemistry, 2nd ed. Little, Brown and Co., Boston.

Pearson, B. 1958 Improvement in the histochemical localization of succinic dehydrogenase by use of nitroneotetrazolium chloride. J. Histochem. Cytochem., 6: 112-121.

Rutenburg, A. M., M. Wolman and A. M. Seligman 1953 Comparative distribution of succinic dehydrogenase in six mammals and modification in the histochemical technic. Ibid., 1: $66-81$.

Scarpelli, D. G., R. Hess and A. G. E. Pearse 1958 The cytochemical localization of oxidative enzymes. I. Diphosphopyridine nucleotide diaphorase and triphosphopyridine nucleotide diaphorase. J. Biophys. Biochem. Cytol., 4: 747-752.

Scarpelli, D. G., and A. G. E. Pearse 1958 Physical and chemical protection of cell constituents and the precise localization of enzymes. J. Histochem. Cytochem., 6: 369-376.

Whitaker, W. L., and B. L. Baker 1948 Inhibition of hair growth by the percutaneous application of certain adrenal cortical preparations. Science, 108: 207-209.

Yielding, K. L., and G. M. Tomkins 1959 Inhibition of the enzymic oxidation of DPNH by steroid hormones. Proc. Nat. Acad. Sci., 45: 1730-1739. 
PLATES 


\section{PLATE 1}

\section{EXPLANATION OF FIGURES}

Abbreviations: A, arrector pili muscle; S, sebaceous gland; C, capillary; P, papilla of hair follicle; F, external sheath of hair follicle.

1 Skin from left nontreated side of neck. PAF fixation, PAS and hematoxylin stain. $\times 110$.

2 Skin from right side of the neck of the rat illustrated in figure 1. Treated with prednisolone for 99 days. The epidermis is somewhat thinner and the dermis is more compact with fewer cells. The panniculus adiposus is wiped out. Technic and magnification as for figure 1.

3 Epidermis from the left nontreated side of a rat which received alcohol for 124 days. $\mathrm{SD}$ is revealed by the disposition of formazan chiefly in the basal cells. $\times 720$.

4 Skin from the left side of a rat which is illustrated also in figure 5. Sebaceous glands (S) are stained intensely with the NSE procedure. Epidermis, external epithelial sheath of the hair follicle (F), arrector pili muscle (A), and scattered cells of the dermis are stained. Note scattered intensely stained cells (arrow) of the epidermis and hair follicle. $\times 120$.

5 Skin from right side of the rat illustrated in figure 4, treated with cortisol for 75 days. In the centrally located sebaceous gland (S) esterase is reduced in the glandular epithelium but remains intense in the secretion at the opening of the gland. Some enzyme remains in follicular sheaths, intensely stained cells are gone from the epidermis and follicle, and the arrector pili muscle (A) is unchanged. The two dense masses at the upper left could not be identified and were unusual. Technic and magnification as for figure 4 .

6 An area of epidermis and dermis from the section illustrated in figure 4. Esterase in the epidermis and some intensely active cells are shown. $\times 240$.

7 An area from the section shown in figure 5. Total esterase is somewhat lower in the epidermis and intensely stained cells are no longer evident. Subepidermal connective tissue cells are less evident. Technic and magnification as for figure 6. 


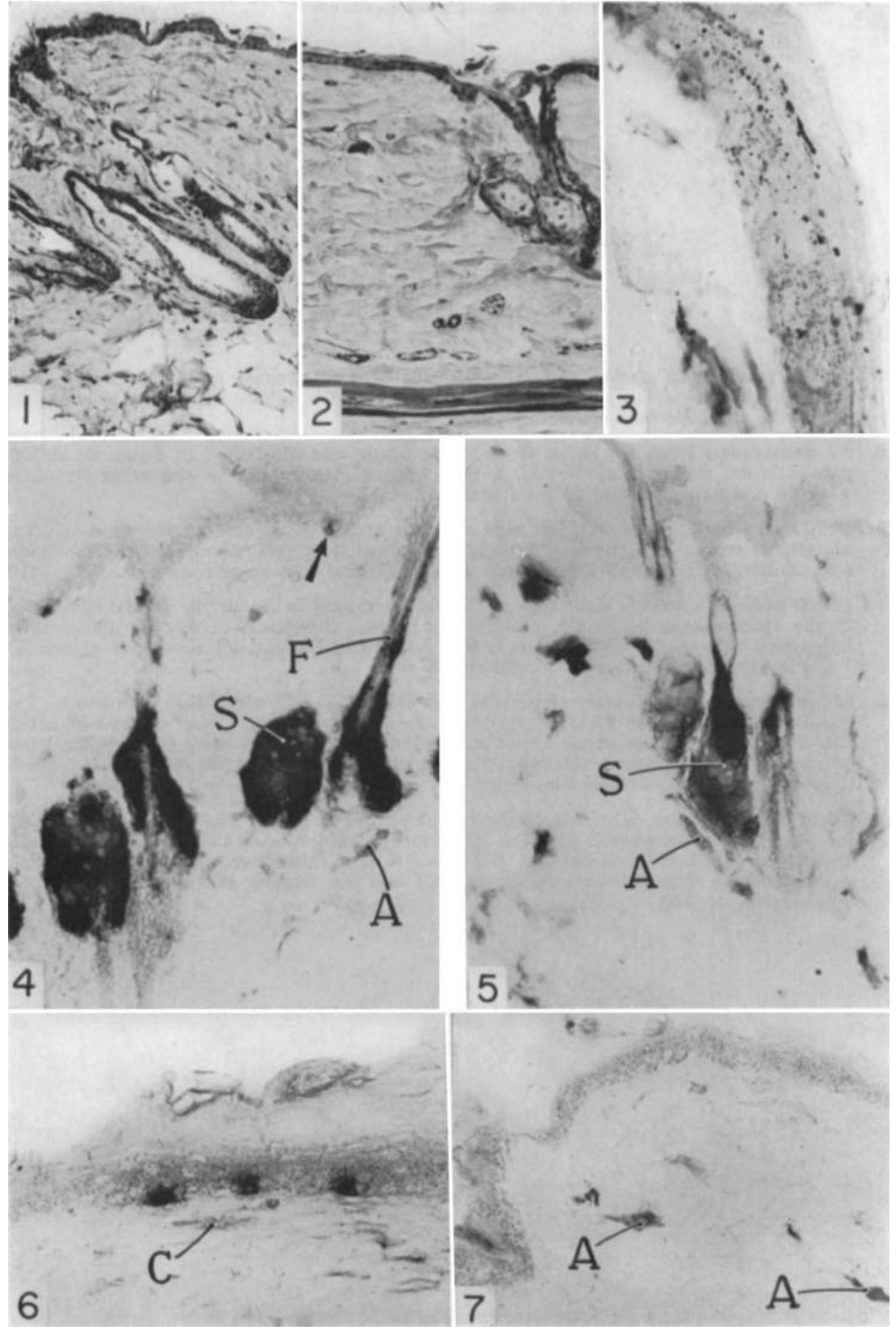




\section{PLATE 2}

EXPLANATION OF FIGURES

8 An AP preparation of skin from the left side of a rat treated for 65 days with prednisolone. Intense activity is revealed in capillaries (C), sebaceous glands (S) and the papilla of the hair follicle $(P) . \times 140$.

9 AP preparation from the right, treated side of the rat illustrated in figure 8 . A hair papilla is not recognizable, but AP is not changed significantly in any other structure. Technic and magnification as for figure 8 .

10 DPND preparation from the left side of a rat treated with cortisol for 78 days. The reaction is strong in epidermis, subepidermal capillaries (C), dermal connective tissue cells, follicular sheaths (F), sebaceous glands (S) and arrector pili muscles (A). $\times 110$.

11 DPND preparation from right side of the rat illustrated in figure 10. DPND is reduced in the epidermis and follicles, and is less evident in dermal connective tissue cells. It remains active in arrector pili muscles (A) and in the smaller sebaceous glands (S). Technic and magnification as for figure 10 .

12 LD preparation of epidermis from the left side of rat illustrated in figure 13. The epidermis is unusually thick because this specimen was taken from an area of active hair growth. In the epidermis LD is most concentrated in the basal cells. Some background staining is present. Mitochondria are stained clearly in the epidermis. Connective tissue cells and dermal capillaries (C) are active. $\times 720$.

13 LD in skin from the right side of the rat illustrated in figure 12, treated with cortisol for 140 days. As compared with the left side, the epidermis is thinner, the basal cells are smaller and less LD is evident. This epidermis is thinner than in areas where hair is not growing. Subepidermal capillaries (C) and the arrector pili muscle (A) remain quite active. $\times 720$. 

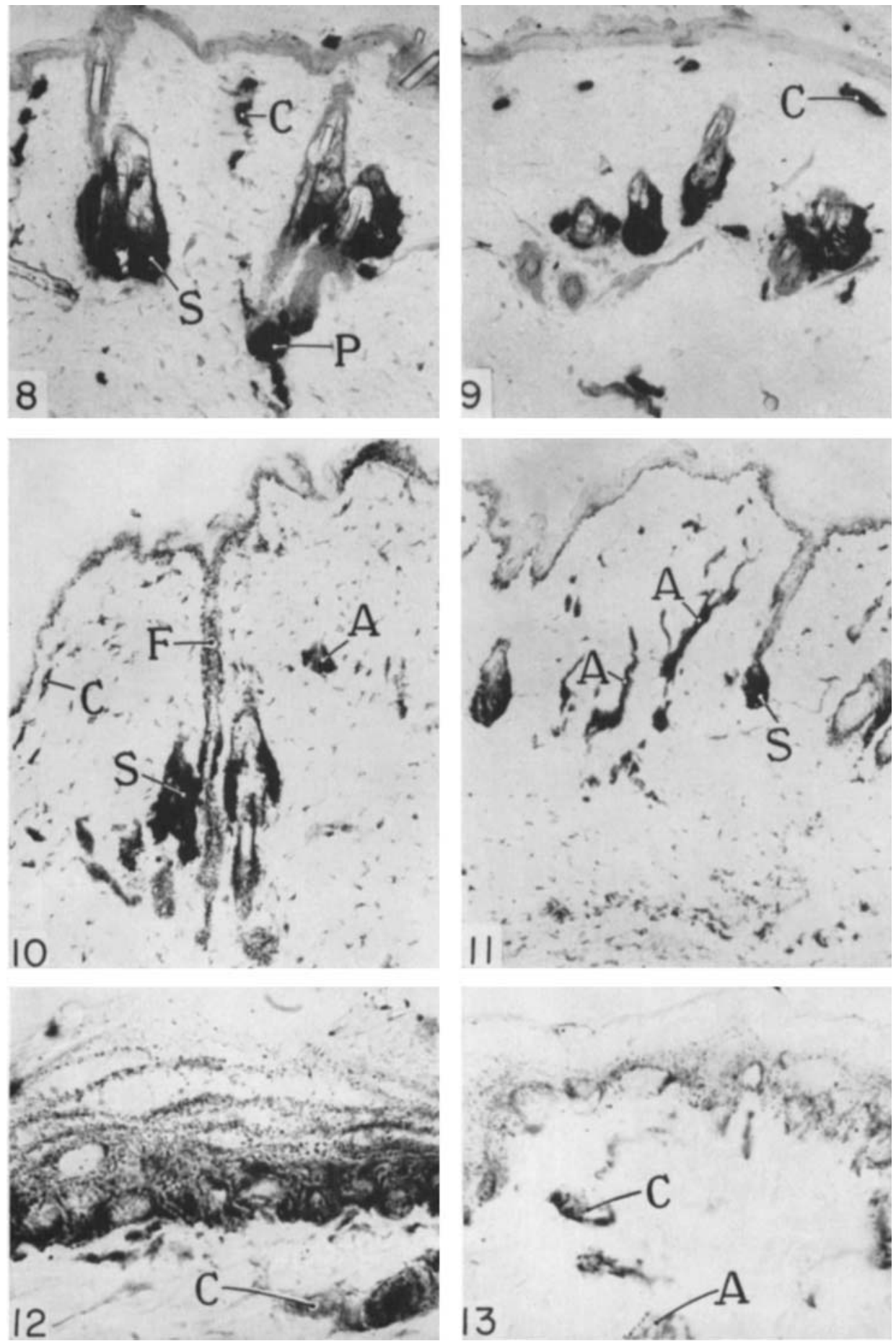